\title{
El comportamiento económico desde la perspectiva biológica y psicológica
}

\author{
Cindy Cifuentes Gómez * \\ Siervo Tulio Delgado Ruiz ** \\ Jorge Iván González ***
}

Fecha de recepción: 10 de febrero de 2021

Fecha de aprobación: 8 de junio de 2021

Resumen: El artículo explora algunos principios del comportamiento económico desde una aproximación biológica y psicológica, mediante un análisis reflexivo sobre la posibilidad de explicar las actuaciones económicas de los individuos a partir de la revisión de aspectos propios de disciplinas que están más allá de las fronteras de las ciencias económicas. Se destaca la importancia que tienen las emociones en la toma de decisiones. La comprensión de estas motivaciones ayuda a mejorar el diseño y la evaluación de las políticas públicas y las decisiones de inversión; además, se muestra que el contexto de desigualdad y felicidad incide en la formación del juicio heurístico.

Palabras clave: comportamiento, economía, biología, psicología, preferencias, creencias, heurística, evolucionismo, adaptabilidad, felicidad, desigualdad.

Clasificación JEL: B59, D11, Y8.

Cómo citar este artículo:

Cifuentes Gómez, C., Delgado Ruiz, S. T., \& González, J. I. (2021). El comportamiento económico desde la perspectiva biológica y psicológica. Apuntes del Cenes, 40(71). Págs. 17 - 43. https://doi.org/10.19053/01203053.v40.n72.2021.12474

\footnotetext{
Magíster en Economía. Investigadora independiente. Tunja, Colombia. cindyc77@hotmail.com (iD) https://orcid.org/0000-0002-6152-4823

** Magíster en Economía. Docente Escuela de Economía, Universidad Pedagógica y Tecnológica de Colombia. Tunja, Colombia. siervo. delgado@uptc.edu.co Dhttps://orcid.org/0000-0001-8120-0909

*** Doctor en Economía. Docente Facultad de Economía, Universidad Externado de Colombia. Bogotá, Colombia. jorgeivangonzalez29@ gmail.com iD https://orcid.org/0000-0003-4845-2690.
} 


\title{
Economic Behaviour from a Biological and Psychological Approach
}

\begin{abstract}
This paper explores some principles of economic behaviour from a biological and psychological perspective, through a reflective analysis on the feasibility of understanding economic actions of individuals from a review of features of non-economic sciences. Emotions are emphasized as important factors in decision-making. Understanding these reasons helps improve the design and evaluation of public policies and investment decisions; furthermore, it is shown that the context of inequality and happiness affects the formation of the heuristic judgment.
\end{abstract}

Keywords: behaviour, economics, biology, psychology, preferences, beliefs, heuristic, evolutionism, adaptability, happiness, inequality. 


\section{INTRODUCCIÓN}

La toma de decisiones económicas no es puramente racional. Aunque este principio ha sido reconocido por los grandes maestros de la disciplina, se ha negado en los libros de texto, que parten del supuesto de un agente racional. Además de las dimensiones valorativas del sujeto, es necesario tener presente la forma en que se toman decisiones en un contexto intertemporal que es incierto. Es importante identificar las relaciones económicas en el marco del evolucionismo biológico darwiniano y mostrar su relevancia en la dinámica del mercado, que es intrínsecamente colectivo. La sociedad se configura a partir de las decisiones individuales, que interactúan en procesos complejos.

El objetivo del presente trabajo, de índole reflexivo, es poner en evidencia que los fenómenos de autotransformación derivan en procesos cognitivos (conscientes e inconscientes), en los que juegan un papel central las emociones y las heurísticas del juicio.
El artículo comienza con una revisión teórica de la economía evolutiva. La segunda parte desarrolla los conceptos de preferencias, racionalidad y creencias desde la lógica biológica. Posteriormente se discute la importancia del enfoque evolutivo, tanto en la psicología como en la economía. Y, finalmente, se examinan los procesos cognitivos y la formación de expectativas de juicios heurísticos.

\section{DELIMITACIÓN CONCEPTUAL}

De acuerdo con Hodgson (1993) y Masera (2013), la historia de la economía evolutiva tiene tres grandes etapas: i) la primera fase corresponde al período que va desde los orígenes de la economía política hasta 1925. Varios autores como Adam Smith, Edgeworth, Carl Menger, Alfred Marshall, entre otros, consideraron relevante el análisis directo de los determinantes psicológicos y biológicos de la decisión humana. ii) Entre 1925 y 1945, la economía le dio mucha relevancia a los modelos de equilibrio estacionario. Pero, al mismo tiempo, el énfasis que le atribuyó Keynes a los "espíritus 
animales" permitió crear las bases de un análisis en el que las decisiones de inversión están muy marcadas por la pasión y el sentimiento. Durante estos años también son relevantes los estudios de Friedrich Hayek sobre el "orden sensorial" y los de Herbert Simon sobre el comportamiento de las empresas. A pesar de los avances mencionados, estos años son considerados la era "oscura" 1 de la economía evolucionista. iii) A partir de 1945 se renuevan los debates sobre los temas evolutivos.
Aunque la corriente evolutiva en economía es muy rica y variada, no todos los autores son por ello "darwinianos". Es evidente que las interpretaciones biologistas analógicas (lado derecho en la Figura 1) u ontológicas (lado izquierdo de las figuras 1 y 2), comparten las metapremisas o los principios más generales del evolucionismo darwiniano. Las diferencias son de intensidad y corresponden al grado de adhesión a este sistema teórico.

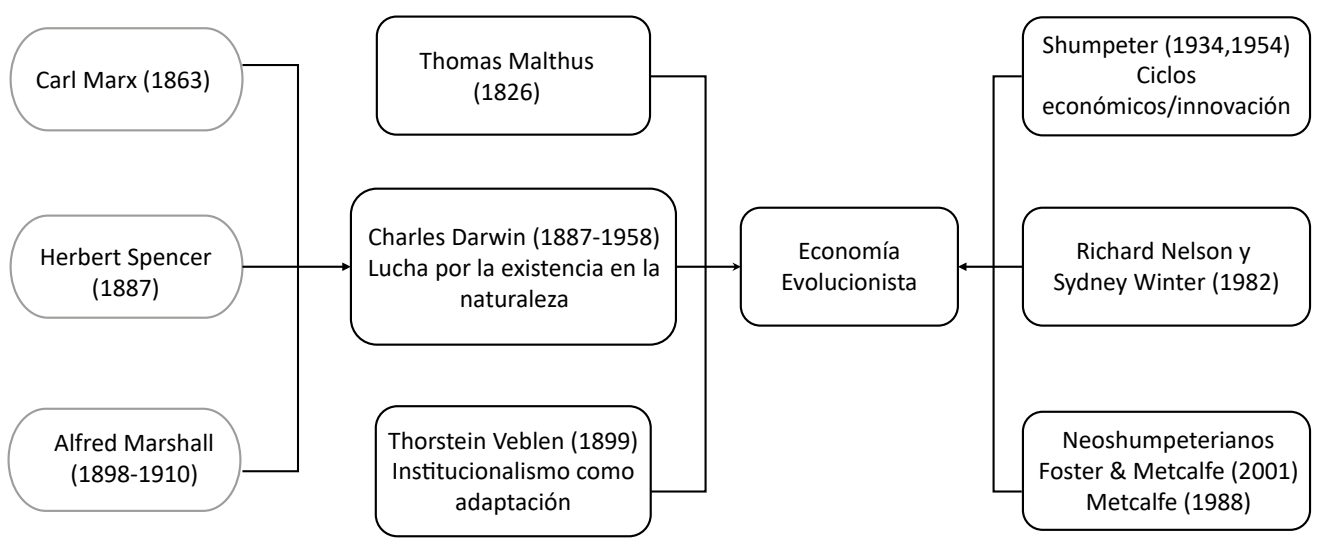

Figura 1. Taxonomía economía evolucionista.

Fuente: elaboración propia.

El enfoque evolucionista analógico facilita la comprensión de los procesos de emergencia y difusión de la innovación tecnológica, y ayuda a explicar el cambio y la adaptación de las organizaciones empresariales. La economía evolucionista ontológica pone su atención en el proceso de transformación de la economía desde su propio interior, porque inscribe su reflexión en la forma como el comportamiento humano impactó el orden institucional (Witt, 2003).

1 El periodo posterior a la II Guerra Mundial es considerado como la "edad oscura" del evolucionismo en las ciencias sociales: "Durante este periodo el evolucionismo fue severamente criticado y llegó a considerarse como una propuesta anticuada, que los investigadores que se respetaran no debían considerar seriamente [...] la palabra 'evolución' llegó a pronunciarse a riesgo de la propia reputación intelectual" (Sanderson, 1990, p. 2, citado en Hogdson, 1997). 
Para Hogdson, el evolucionismo es, principalmente, una visión del mundo muy amplia e inclusiva que contribuye a la explicación de las dinámicas sociales.
En su opinión, el darwinismo contiene un amplio sistema teórico que va más allá de la biología (Hogdson, 1997).

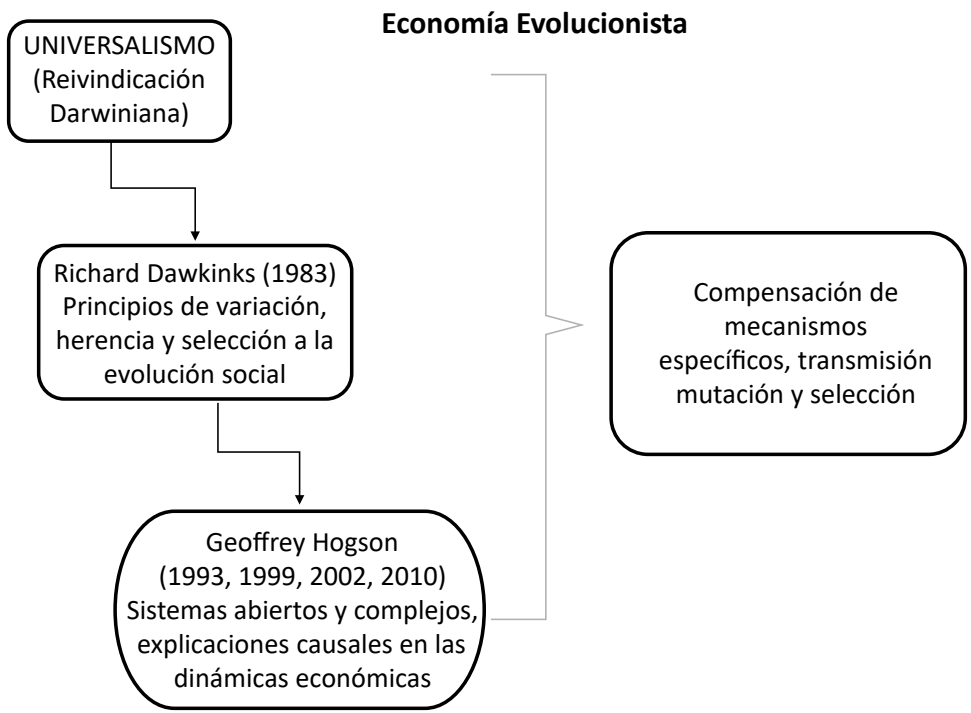

Figura 2. Universalismo, reivindicación darwiniana.

Fuente: elaboración propia.

Gracias al pensamiento evolutivo, es posible reflexionar de manera sistemática sobre categorías como organismo social, población, competencia, evolución, adaptación (La Rocca, 2003). Desde la biología adquiere relevancia el sujeto. Por esta razón, Mantzavinos et al. (2004) sugieren que la mente es como un complejo estructural que interpreta activamente y, al mismo tiempo, clasifica las variadas señales recibidas por los sentidos. La mente ordena las experiencias del entorno físico y del sociocultural-lingüístico:
El proceso de cambio social que presentamos en este artículo se puede resumir de la siguiente manera: "Realidad"> creencias $>$ instituciones $>$ políticas específicas $>$ resultados (y, por lo tanto, "realidad" alterada). El mecanismo de retroalimentación de los resultados a la realidad se ejecuta a través de la mente humana; ya que la mente interpreta la realidad, tenemos un conocimiento muy limitado de cómo los resultados serán percibidos e interpretados por los agentes. Esta es la razón principal por la cual los modelos mecanicistas y deterministas del cambio no pueden funcionar: las ideas son los factores autónomos de la evolución socioeco- 
nómica, y si queremos aprender más sobre este proceso, necesitamos saber más sobre la forma en que las mentes construyen la realidad ${ }^{2}$. (Mantzavinos et al., 2004, p. 80)

Los trabajos de Tversky y Kahneman (1986) son especialmente relevantes en este sentido. El examen del comportamiento humano es fundamental para entender el quehacer económico. La felicidad y la función de utilidad adquieren nuevas dimensiones, porque ya no se pueden estandarizar alrededor de un agente representativo. El hedonismo recupera su individualidad. Para Tversky y Kahneman (1991), no hay sujetos homogéneos. La concepción racional de las personas no es totalizante, porque los sentimientos y las emociones condicionan la decisión (González, 2011).

\section{Biología, preferencias, racionalidad y creencias}

El acercamiento de la economía hacia la neurociencia y la psicología ha sido afortunado, ya que ha permitido iluminar las causas próximas del comportamiento. La racionalidad se ha definido como la capacidad que tiene un individuo de maximizar la utilidad, utilizando toda la información disponible, dadas unas restricciones contextuales (ingreso, distancia, etc.) y unas preferencias y creencias (Robson, 2003). Simon (1986) considera que en econo- mía la racionalidad hace énfasis en el acto de elegir, mientras que en otras disciplinas se destacan los aspectos procedimentales. Entre las dimensiones contextuales que determinan la elección es necesario tener en cuenta las preferencias preexistentes (Flores \& Ostroksy-Solís, 2008 ).

La utilidad tiene un contenido emocional o hedónico. Este postulado básico ya había sido enunciado por Bentham y Edgeworth. En la literatura contemporánea, Damasio (1994) muestra que las emociones son intrínsecas a cualquier decisión racional (Bechara et al., 1997; Robson, 2002; Robson, 2003; Robson, 2011). La respuesta emocional está presente en cualquier decisión. En la economía experimental se han realizado ejercicios que muestran que un determinante de la utilidad es la tensión recompensa/penalización (Robson, 2003). Para Ng (1996), la racionalidad persigue una maximización flexible impulsada por la recompensa y la penalización.

El examen del proceso de elección es más complejo cuando se introduce el aprendizaje (Bergman, 1998), que se va configurando mediante un "refuerzo" apropiado a medida que se van conociendo las consecuencias de las acciones. En la elección también importa la interacción entre los logros intermedios y las metas finales. Esta secuencia intertemporal es determi-

2 Traducción propia. 
nante, porque el objetivo último se va reconfigurando al mismo tiempo que se modifican los elementos contextuales.

\section{Naturaleza adaptable $y$ utilidad hedónica}

Tremblay y Schultz (1998) examinan la forma como las preferencias se van adaptando a la experiencia. Suponga las preferencias sobre tres recompensas. A es preferida a $B$ y $B$ es preferida a $C$. Por transitividad, $A$ es preferida a $C$. Estas recompensas se presentan en pares, $\mathrm{y}$ las señales se asocian con cada recompensa. Si $B$ y $C$ están involucrados, la señal asociada con $B$ hace que muchas de estas neuronas sean más activas que la señal asociada con $C$. Si $A$ y $B$ están involucrados, $A$ induce una actividad neurológica similar a $B$ en la situación de primera elección, y $B$ tiene un efecto similar a $C$. Los autores llegan a la conclusión de que la actividad neuronal no está ligada a las recompensas en sí mismas, sino a la escogencia de la mejor de las dos opciones ${ }^{3}$.

La preferencia está marcada por el sujeto y su contexto, y la persona la va modificando dependiendo de las circunstancias. Por esta razón, no tiene mucho sentido afirmar que las preferencias se mantienen a lo largo del tiempo. En los modelos de estado estacionario este es uno de los principales supuestos.

\section{Utilidad adaptativa a corto plazo}

Junto con los reportes anuales de felicidad mundial (WHR por sus siglas en inglés), en otros estudios también se les pide a las personas que estimen su propio nivel de felicidad. Las respuestas guardan una estrecha relación con el rango que el individuo ocupa en la sociedad (Scitovsky, 1992; Oswald, 1997). Si pertenece a un grupo de clase media, su percepción de la felicidad es similar a la que tenía la generación anterior, que también hacía parte de la clase media. Esta simetría se presenta, aunque los niveles de consumo y las perspectivas de vida sean muy diferentes. De manera similar, el individuo medio en un país pobre tendría un nivel de utilidad similar al individuo medio en un país rico.

Si la felicidad está asociada a la riqueza, la persona de altos ingresos siempre aumenta su felicidad, pero a un ritmo menor. Y la persona pobre, aún con variaciones mínimas del ingreso, aumentará significativamente su percepción de felicidad, tal como se aprecia en la Figura 3. Ante los mismos cambios marginales de la riqueza, la felicidad del pobre aumenta más que la del rico.

\footnotetext{
3 Ver, además, Watanabe y Sakagami (2007).
} 


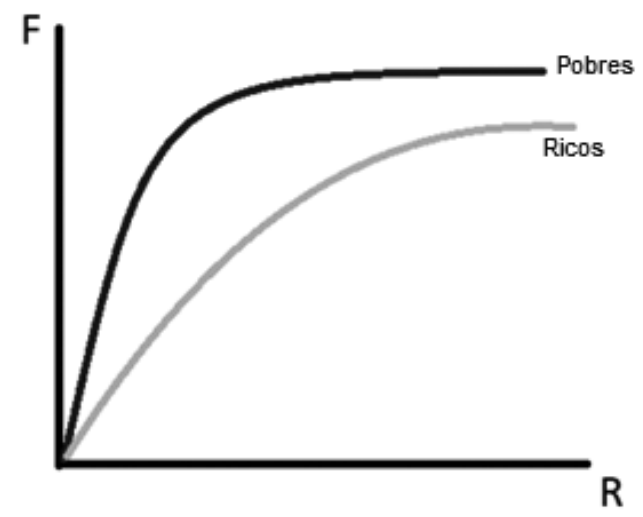

Nota: el eje horizontal corresponde a los cambios marginales de la riqueza y el vertical a la felicidad.

Figura 3. Percepción de felicidad vs. riqueza. Cambios marginales.

Fuente: elaboración propia.

La persona tiene una escala subjetiva de lo bueno y lo malo. La situación actual es mala (buena) si está por debajo (encima) del umbral. Las categorías de lo bueno y lo malo, y del nivel del umbral no son fijas, sino que se van moviendo. La percepción se modifica porque varía el contexto, o porque la dimensión temporal cambia. Y la visión que en determinado momento se tiene del futuro se expresa en el consumo (Robson, 2002; Rogers, 1994). La Figura 4 ilustra esta idea.

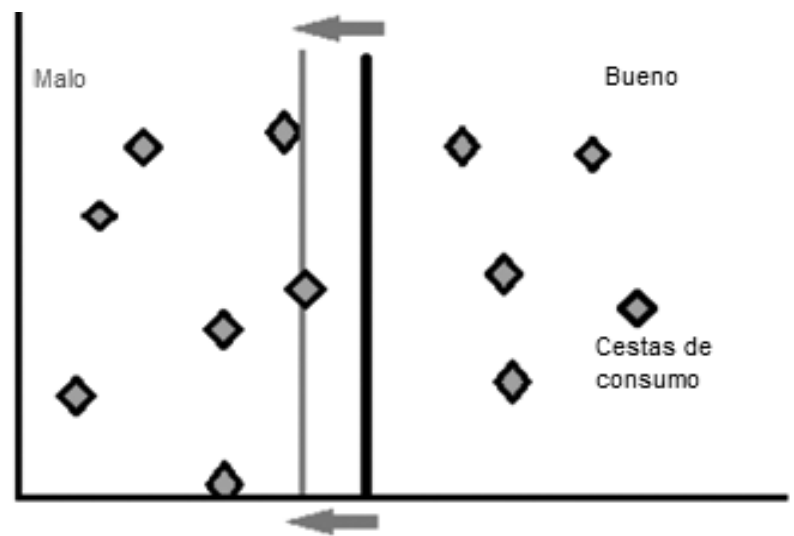

Desplazamiento del

Umbral

Figura 4. Adaptación de la elección frente a desplazamientos del umbral óptimo de elección.

Fuente: elaboración propia. 
Para que la tasa de preferencia intertemporal no cambie a lo largo del tiempo, se debe suponer que los aspectos contextuales no se modifican y, por tanto, no hay desplazamientos del umbral (Loewenstein \& Prelec, 1992). Esta forma de estabilidad es un supuesto muy exigente. En la realidad, el individuo tiene preferencias "inconsistentes en el tiempo" (Strotz, 1956, p. 171)4.

\section{El enfoque evolutivo de la psicología económica}

Darwin, que mantuvo un estrecho diálogo con Marshall, trata de entender el análisis de los procesos psicológicos que subyacen a la conducta humana. En el libro La expresión de las emociones en el hombre y los animales busca, a partir de observaciones y comparaciones, entender las especificidades de la conducta humana (Darwin, 1872). Estos aportes se han tratado de concretar en la llamada psicología evolucionista del desarrollo (PED):

La aplicación de los principios básicos de la evolución darwinista, en particular la selección natural, sirven para explicar el desarrollo humano contemporáneo. Implica el estudio de los mecanismos genéticos y ambientales que subyacen al desarrollo universal de las competencias cognitivas y sociales, así como de los procesos epigenéticos (interacciones gen-ambiente) evolucionados que adaptan dichas competencias a las condiciones locales; asume que no solo los comportamientos y las cogniciones que caracterizan a los adultos son producto de las presiones de la selección actuando en el curso de la evolución, sino también las características de los comportamientos y de las mentes infantiles. (Bjorklund \& Pellegrini, 2002, p. 4)

La psicología evolucionista emerge como disciplina académica formal solo a finales de los años 80 y principios de los 90. Con el paso del tiempo se han ido perfeccionando los programas de procesamiento de información (Hernández-Blasi et al., 2003). Los programas cognitivos evolucionados dan origen al pensamiento y el comportamiento adaptativo (Geary \& Huffman, 2002; Bjorklund, 2000; Fodor, 2000; Sternberg, 1997).

Dolan et al. (2012) recopilan los nueve efectos más robustos que influyen en el comportamiento humano de manera automática. Los autores hacen una "revisión integradora" de la literatura utilizando el marco mnemotécnico Mindspace, lo que ellos describen como un conjunto de herramientas o lista de verificación para los responsables de políticas públicas y con la capacidad de influir en las decisiones colectivas.

Mindspace es un intento de categorizar la literatura. La tabla 1 resume los elementos, los cuales serán desarrollados a lo largo de este apartado.

$4 \quad$ Ver: McClure, Laibson, Loewenstein y Cohen (2004); McClure, Ericson, Laibson, Loewenstein y Cohen (2007). 
Tabla 1. El marco de Mindspace para el cambio de comportamiento

\begin{tabular}{cc}
\hline Mindspace & Comportamiento \\
\hline $\begin{array}{c}\text { Mensajero } \\
\text { Incentivos } \\
\text { Normas }\end{array}$ & Estamos influenciados por quien nos comunica información \\
Predeterminados & Estamos fuertemente influenciados por lo que hacen los demás \\
Atención & "Vamos con el flujo" de opciones preestablecidas \\
Accesibilidad & Nuestra atención se centra en lo que es novedoso y nos parece relevante \\
Afecto & Nuestros actos son a menudo influenciados por señales subconscientes \\
Compromisos & Nuestras asociaciones emocionales pueden moldear nuestras acciones \\
Ego & Buscamos ser coherentes con nuestras promesas públicas y con actos recíprocos \\
\hline
\end{tabular}

Fuente: tomado de Dolan et al. (2012)

Los procesos de decisión se pueden analizar desde dos perspectivas, dependiendo de si el énfasis se pone en la teoría conductual o en la cognitiva. La dimensión psicológica ha ido ganando relevancia en el análisis económico, y una muestra de ello es el Premio Nobel que se le otorgó en el 2002 a Daniel Kahneman y a Vernon Smith "[...] por integrar aspectos de la teoría psicológica sobre el comportamiento económico del ser humano en momentos de incertidumbre y realizar análisis empíricos de laboratorio, especialmente sobre mecanismos alternativos de mercado" (The Nobel Prize, 2021), y en 2017 se le concedió el Nobel a Richard Thaler "[...] por sus contribuciones a la economía conductual" (The Nobel Prize, 2021).
Estos análisis que enfatizan el papel de la psicología de las decisiones suponen, de entrada, que la racionalidad es limitada. Se podrían destacar tres enfoques. El primero explora la heurística del juicio (Kahneman \& Tversky, 1982). El segundo se centra en la teoría prospectiva (Kahneman \& Tversky, 1979; Tversky \& Kahneman, 1991). La tercera línea de investigación se ocupa de los efectos marco, framing effects, y de sus implicaciones para los modelos del agente racional (Tversky \& Kahneman, 1981; Tversky \& Kahneman, 1986).

\section{Heurística del juicio}

Las ideas subyacentes son: (i) la mayor parte de los juicios y de las elecciones se efectúan intuitivamente; (ii) las reglas que gobiernan la intuición son generalmente similares a las de la percepción (Kahneman \& Frederick, 2005). 
En este análisis se diferencian dos modos de pensar y decidir que, a grandes rasgos, corresponden a los conceptos habituales de razonamiento intuitivo 5 .

El razonamiento se hace deliberadamente y con mucho esfuerzo, mientras que el pensamiento intuitivo parece que se presenta de forma espontánea en la mente, sin cálculo o búsqueda consciente, y sin esfuerzo. La observación superficial y la investigación sistemática indican que la mayor parte de los pensamientos y las acciones son normalmente intuitivas en este sentido. (Gilbert, 1989, 2002; Wilson, 2002; Epstein, 2003; citados en Kahneman, 2002, p. 183)

El individuo usualmente confía en el juicio que le viene rápidamente a la cabeza. Cuando se tiene un conocimiento anterior, que ha sido conseguido de manera sistemática, el juicio intuitivo puede ser bastante acertado. El arquitecto tiene una noción del espacio más clara que un biólogo y puede calcular de manera intuitiva y con cierta precisión el área de un cuarto (Kahneman \& Frederick, 2005).

Kahneman (2003) muestra que el sistema cognitivo es un mecanismo computacional que se adapta bien a su entorno y cuenta con dos sistemas de ajuste: un proceso a corto plazo, que es flexible, y uno a largo plazo, que consiste en la adquisición de destrezas que al final generan respuestas muy eficaces con costes reducidos. Esta óptica de análisis difiere en aspectos importantes del paradigma del agente racional planteado en la teoría económica. El marco de Mindspace se centra en los impulsores de comportamiento más automáticos (Sistema 1) (Dolan et al., 2012).

\section{Teoría prospectiva}

En el análisis económico estándar se supone que las decisiones dependen del estado final de la dotación. Desde otra mirada, en el contexto de las elecciones con riesgo se introduce la noción de utilidad esperada, ya que el resultado final nunca se conoce con certeza (Bernoulli, 1954). Para Kahneman y Tversky (1982), el análisis de la utilidad tiene que considerar de manera explícita la situación inicial, que determina la percepción sobre el cambio. La persona A, que experimenta una reducción de la riqueza de cuatro millones de pesos a tres millones, se siente peor que otro individuo $\mathrm{B}$, que aumenta su riqueza de un millón de pesos a un millón cien mil pesos 6 .

\footnotetext{
$5 \quad$ Respecto a las características que diferencian los dos tipos de procesos cognitivos, Stanovich y West (2002) propusieron los rótulos neutrales de Sistema 1 y Sistema 2. Las operaciones del Sistema 1 son rápidas, automáticas, sin esfuerzo, asociativas y a menudo están cargadas emocionalmente. Además, vienen determinadas por la costumbre y consecuentemente son difíciles de controlar o modificar. Las operaciones del Sistema 2 son más lentas, consecutivas, requieren un gran esfuerzo y están controladas de forma deliberada; son también relativamente flexibles y, potencialmente, vienen determinadas por reglas.

6 Para el análisis sobre las interacciones neuronales ocasionadas por una pérdida, se puede consultar en Tom et al. (2007) y De Martino et al. (2006).
} 
La teoría prospectiva se ha presentado como una alternativa fáctica y no como un postulado normativo. La Figura 5 muestra que el comportamiento del individuo es muy diferente cuando hay ganancias o pérdidas. Mientras que la valoración de las ganancias se reduce en el margen, el de las pérdidas puede generar círculos viciosos endógenos. A diferencia de la dinámica que se observa en la figura, en Bentham la felicidad y el dolor tienen comportamientos simétricos.
De manera más específica, la curva tiene estas características:

(1) Ser cóncava en el campo de las ganancias, con lo que propicia la aversión al riesgo; (2) ser convexa en el dominio de las pérdidas, favoreciendo así la búsqueda de riesgos; (3) lo más importante, es una función que se quiebra bruscamente en el punto de referencia, y con aversión a las pérdidas -más empinada para las pérdidas que para las ganancias. (Kahneman, 2002, p. 193)

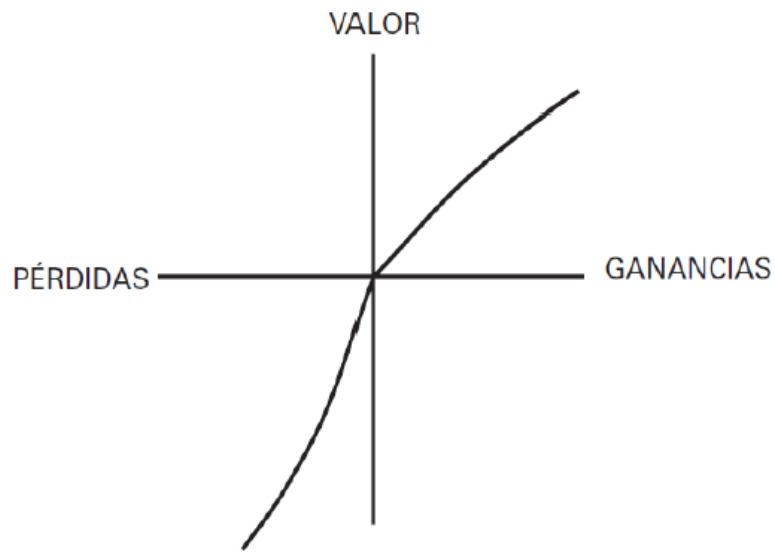

Figura 5. Función de valor esquemática de los cambios.

Fuente: tomada de Kahneman (2002)

El efecto dotación, endowment effect en inglés, es un sesgo cognitivo que describe el valor inconsciente que da un individuo a sus posesiones. En la teoría del consumidor existe un "efecto dotación": el precio de venta es mucho más elevado que el precio de compra, a menudo se multiplica por un factor de dos o más.
Kahneman y Tversky (1974) proponen una teoría alternativa para el riesgo, en la que la utilidad se vincula a las ganancias y a las pérdidas -variaciones de la riqueza en vez de niveles de esta. Entonces, la utilidad no se puede separar de la emoción y esta depende de las circunstancias (Kahneman,1994, 
2000). Además, la aversión a las pérdidas juega un papel importante en el análisis económico (Thaler, 1980).

Las personas odian las pérdidas. En términos generales, es dos veces mayor la desgracia de perder un cierto ingreso, que la alegría de ganar un monto similar (Thaler \& Sunstein, 2017). Así que el valor del bien parece ser más elevado cuando se pierde que cuando se gana (Thaler \& Kahneman, 1991).

\section{Efectos marco}

Un supuesto poco realista del modelo del agente racional es que los agentes efectúan sus elecciones en un contexto que incorpora todos los detalles relevantes de la situación. Pero, en realidad, la decisión se toma en situaciones de "marcos limitados" (narrow framing) (Kahneman \& Lovallo, 1993; Thaler, 1985, 1999; Arrow, 1982; Tversky \& Kahneman, 1986). La invariancia no se cumple cuando se presentan los efectos marco (Tversky \& Kahneman, 1981)7.

\section{Heurística del juicio}

Dada su relevancia, es oportuno profundizar en las características de la heurística del juicio. Las extensiones recientes del concepto de la heurística en el campo del afecto pueden ser de especial trascendencia para el diálogo entre la psicología y la economía.
Para Rottenstreich y Hsee (2001), los individuos prefieren resultados cargados emocionalmente, que los logros de tipo monetario. El concepto de heurística de los afectos explica que el afecto (agradar o desagradar) influye en procesos que se podrían considerar "racionales" y "técnicos" (Slovic, 2002). Loewenstein et al. (2001) justificaron la proposición de que las creencias acerca de los riesgos son, a menudo, expresiones de las emociones, que no se ajustan a la lógica de las preferencias económicas. Las decisiones que se toman en el Sistema 1 no concuerdan necesariamente con las del Sistema 2.

Kahneman (2002) utiliza la heurística de representatividad para explicar sesgos sistemáticos en las valoraciones realizadas bajo incertidumbre. Se refiere, por ejemplo, a las predicciones no regresivas, al olvido de la información, al exceso de confianza y sobrevaloración de la frecuencia de acontecimientos que se recuerdan con facilidad. Estos comportamientos cabrían dentro de las heurísticas del prototipo (Posner \& Keele, 1968; Rosch $\&$ Mervis, 1975).

El prototipo de un conjunto viene caracterizado por los valores medios de las propiedades más notables. La gran accesibilidad de la información del prototipo realiza una función adaptativa importante. Permite que los estímulos nuevos sean clasificados eficientemente, mediante la comparación de sus

7 Sobre las diversas modalidades de riesgo, ver Lichtenstein et al. (1978) y Kusev et al. (2009). 
rasgos con los de los prototipos de la categoría. (Kahneman, 2002, p. 206)

Experimentos de campo (e incluso la prensa diaria) muestran que la heurística del prototipo termina generando errores importantes y sistemáticos en las decisiones económicas (Genesove, 2001). Y una vez que se ha caído en la dinámica del error, los incentivos no son suficientes para contrarrestar la percepción (Lerner, 1999). Y lo que es normal e intuitivo en una determinada situación, no lo es en otras circunstancias (Ross \& Nisbett, 1991).

Con el fin de contextualizar lo anterior, veamos los resultados que arrojan los reportes de felicidad. La Figura 6 es tomada de World Happiness Report (2017), en donde se clasifica a 155 países según sus niveles de felicidad.

La satisfacción con la vida se obtiene de la respuesta a la siguiente pregunta: "Por favor, imagine una escalera, con pasos numerados del 0 al 10 en la parte superior. La parte superior de la escalera representa la mejor vida posible para usted y la parte inferior de la esca- lera representa la peor vida posible para usted. ¿En qué escalón diría que personalmente se siente en este momento?

En la Figura 7 se observan los reportes de felicidad de algunos países, en donde Colombia y México aducen un nivel de satisfacción similar a países como Australia e incluso están por encima de Rusia.

Es notoria la paradoja en donde a pesar de las limitaciones objetivas, la percepción de felicidad de los individuos puede ser muy buena. Este tipo de situación se observa, por ejemplo, en varios países de América Latina, con altos niveles de desigualdad, corrupción, victimización, dificultades económicas e irrespeto a las normas sociales8.

En el ranking mundial, Colombia ocupa el puesto 36, aun así, en el 2017 la pobreza multidimensional tuvo una incidencia del $17 \%$. En las cabeceras municipales la incidencia fue del $11.4 \%$ y en los centros poblados y rural disperso, del $36.6 \%$ (Departamento Administrativo Nacional de Estadística [DANE], 2017).

$8 \quad$ Las normas sociales influyen en el comportamiento, porque los individuos se inspiran en lo que hacen los demás y utilizan sus percepciones de las normas como un estándar para comparar sus propios actos. El funcionamiento de las normas sociales es, al menos en parte, consciente. La conformidad puede ser una estrategia deliberada, ya que podemos obtener placer al elegir comportarnos como todos los demás (Clapp \& McDonnell, 2000). 


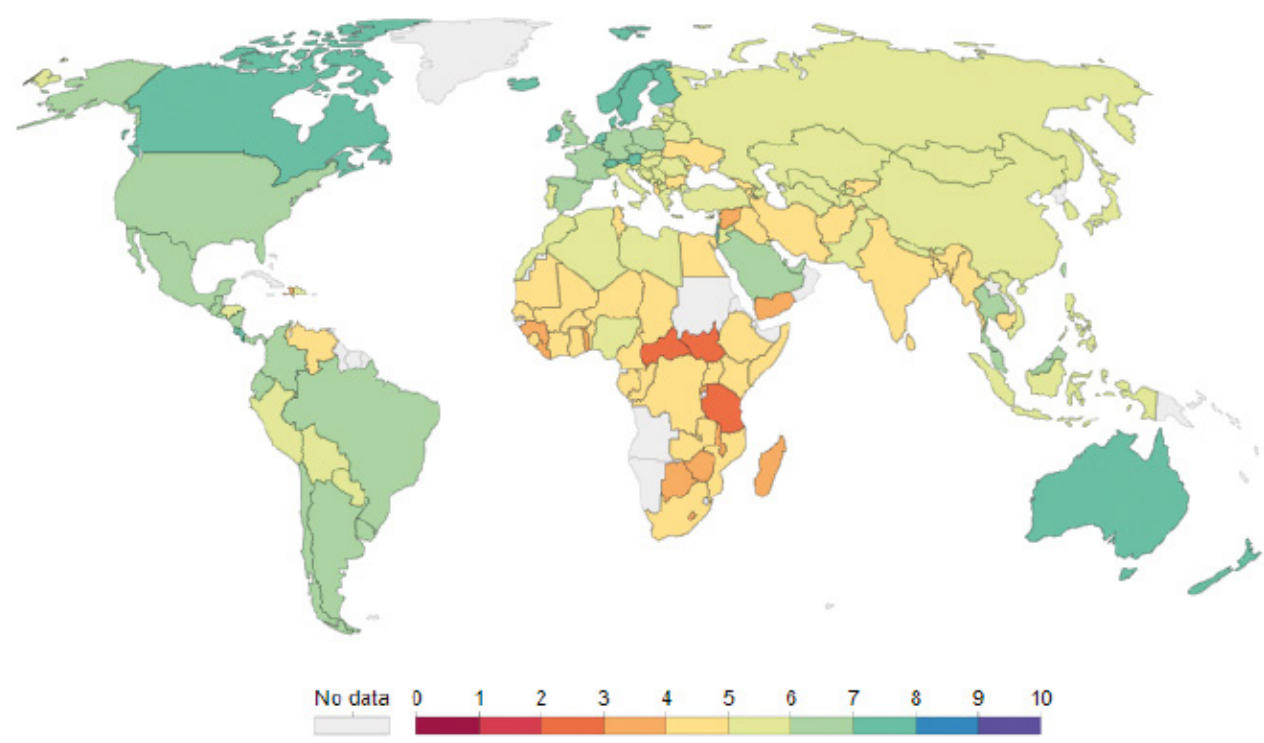

Figura 6. Satisfacción de vida autorreportada.

Fuente: tomada de: World Happiness Report (2017), Recurso: GallupWorldPolls, 2006-2016.

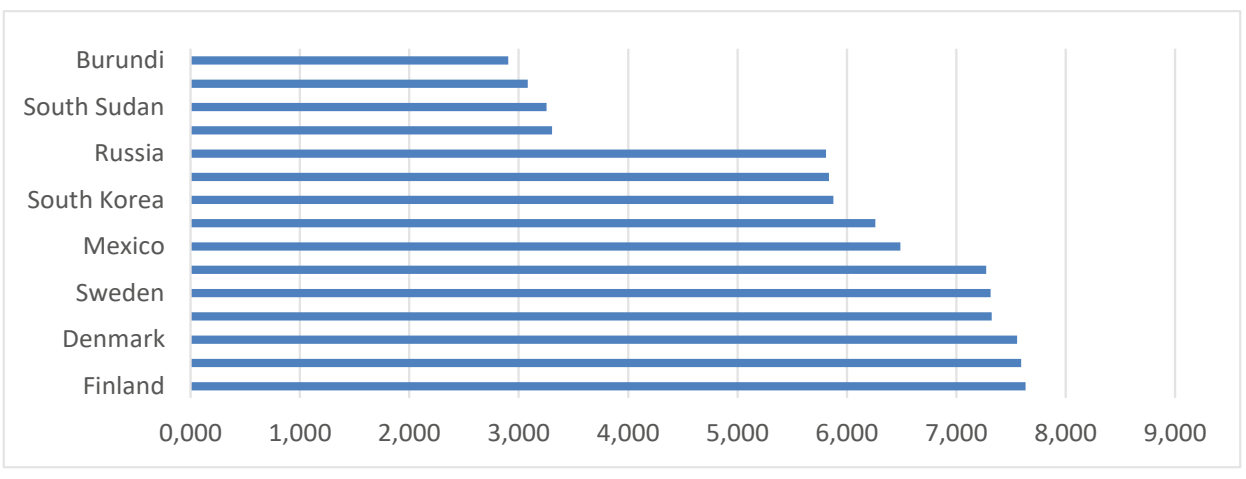

Figura 7. Ranking de felicidad (2017).

Fuente: elaboración propia

Hernández (2011) cita a Lora una elevada desigualdad, está alejada (2008) sobre el estudio del Banco de la realidad, o se trata de personas Interamericano de Desarrollo, en el "resignadas", con un cierto nivel de que se muestra que la población de contentamiento ante sus condiciones los países que son felices a pesar de adversas. Según el autor: 
[...] en este contexto, un pueblo no es fiable, ni sus votaciones ni las opiniones acerca de ellos mismos, debido a la alta brecha entre realidad y su percepción. Se trata de una inconsistencia que los psicólogos llaman adaptación e impulso respuesta, mientras que otras disciplinas de las ciencias sociales como la sociología y la economía política le denominan conciencia falsa, y se le relaciona con conceptos como el de poder. En economía se lidia también con términos como comportamiento irracional, falla de mercado o asimetría de información. (Ungar, 2005, citado en Hernández, 2011) ${ }^{9}$

La felicidad (o la búsqueda de esta) se redefine en términos de adaptabilidad y consistencia, y no puramente en realización de logros. Según Hernández (2010), la felicidad de los individuos dependería:

$$
\text { dFelicidad } / \partial_{t}=\frac{\partial \text { Heurística } / \partial_{t}}{\partial \text { Realización } / \partial_{t}} \quad \begin{aligned}
& \text { Tanto de su capacidad de adaptarse al entorno (heurística o } \\
& \text { felicidad "sintetizada") como del cumplimiento o no de sus } \\
& \text { expectativas (realización o felicidad natural). }
\end{aligned}
$$

A su vez, la evolución de la heurística depende de los logros realizados, así no hayan sido metas preconcebidas, frente a la adversidad del entorno, y la evolución de la realización depende también de los logros realizados (como metas preconcebidas), pero frente a las expectativas propias.

$$
\begin{gathered}
\text { dFelicidad } / \partial_{t}=\frac{\partial \text { Expectativas } / \partial_{t}}{\partial \text { Adversidad } / \partial_{t}} \\
\int \frac{\partial \text { Adversidad }}{\partial_{t}}-\frac{\partial \text { Expectativas }}{\partial_{t}}
\end{gathered}
$$

De allí se obtiene la evolución de la felicidad en términos de la evolución de las expectativas y la adversidad (por ejemplo, crisis) independientes de logros (preconcebidos o no) en el tiempo.

La brecha entre expectativas y adversidad se determina de la siguiente forma, finalmente esta es la que conlleva cambios en las expectativas de los agentes.

Figura 8. Felicidad sintetizada y real: heurística y realización.

Fuente: elaboración propia con base en Hernández (2010).

Los países con mayor desigualdad pueden tener una adaptabilidad que les permite "contrarrestar" de manera subjetiva sus condiciones objetivas. En la Figura 9 se muestra la relación entre desigualdad y percepción de la felicidad. No hay una relación directa entre la dinámica del Gini y los cambios en la satisfacción. De todas maneras, dada la alta desigualdad, el nivel de satisfacción es relativamente favorable. Sin duda, esta manera de ver la felicidad está asociada a bajas aspiraciones y a expectativas y metas fácilmente realizables.

9 De acuerdo con el marco de Mindspace, este resultado podría explicarse por el peso que los individuos le dan a la información en función de la percepción que se tenga del "mensajero" (Dolan et al., 2012). 


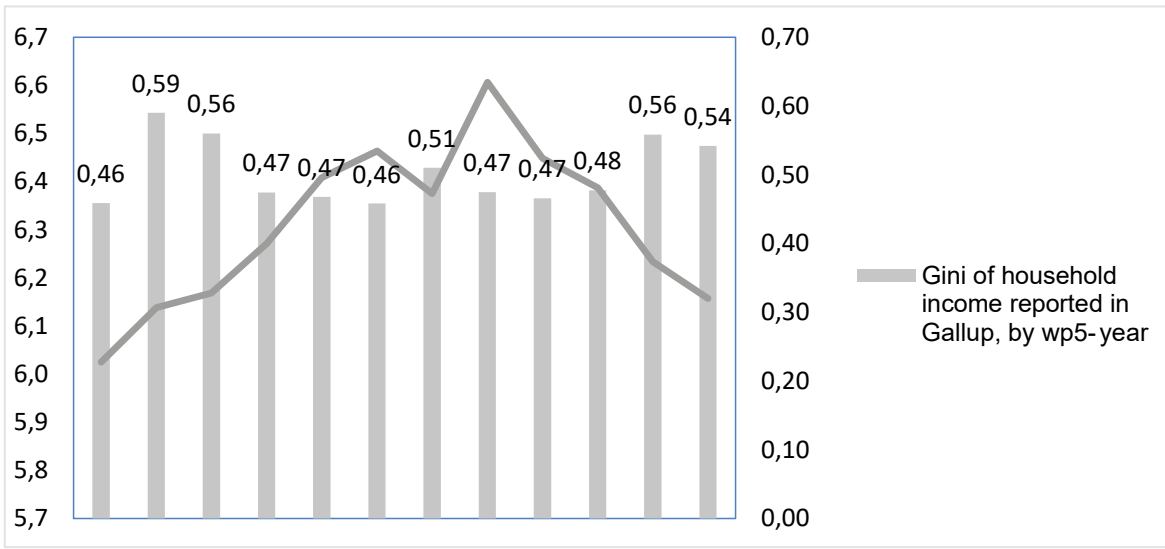

Figura 9. La satisfacción de vida y el Gini del ingreso familiar en Colombia (2006-2017).

Fuente: elaboración propia a partir de la base de datos de World Happiness Report (2018).

Las intervenciones tradicionales que apuntan a "cambiar las mentes" a través de la educación y la información, generalmente funcionan mejor para los países más educados e informados ${ }^{10}$.

Entre los sesgos presentes en la literatura científica se destacan:

- El sesgo de sobreconfianza (overconfidence). Los seres humanos tienden a sobrevalorar sus habilidades, conocimientos e imaginarios de futuro (Camerer \& Lovallo, 1999). Se creen mejores pronosticadores de lo que realmente demuestra la evidencia empírica. La sobreconfianza ha sido estudiada por sus implicaciones en las decisiones económicas. Según Camerer (1996), el sesgo persiste en el tiempo sin descender en intensidad. Este optimismo subjetivo se refleja, por ejemplo, en el empresario que insiste en la rentabilidad de su negocio, en contra de los resultados financieros (Montoya, 2010). Los inversionistas tienden a sobreestimar los rendimientos futuros y a subestimar los resultados negativos.

- El sesgo de excesivo pesimismo. En psicología, el excesivo pesimismo refiere a un procesamiento cognitivo que opera como una elevada tendencia a subestimar los resultados positivos o favorables y al mismo tiempo a sobreestimar los resultados desfavorables o considerarlos muy frecuentes.

Las conclusiones no se desprenden de la experiencia previa ni de cálculos estadísticos, sino que encierran creencias basadas en expectativas y en deseos del decisor.

10 La información sobre cómo acceder a los programas para dejar de fumar, por ejemplo, ha tenido el mayor efecto en los fumadores más adinerados y, por lo tanto, ha ampliado la brecha social entre los más sanos y los menos sanos (Schaap, 2008). 
En el caso de Colombia este sesgo proviene, en gran medida, de la falta de credibilidad que tienen los individuos en las instituciones sociales, democráticas, electorales. También influye la desconfianza interpersonal. Según Lagos (2016), ocho de cada diez ciudadanos no confían en el "otro".

- El heurístico de afecto muestra la forma como las emociones impactan las decisiones (Camerer et al., 2005). Este punto es importante, ya que demuestra que los individuos de la región relacionan en buena parte el concepto de felicidad a las interacciones sociales con los demás.

La Tabla 2 muestra los resultados de Encuesta Mundial de Gallup y que están asociadas al afecto positivo' ${ }^{11}$. La situación: ocho de los diez primeros países en el mundo son de América Latina. Los países no latinoamericanos en los diez primeros son Canadá y Filipinas.

Tabla 2. Los 15 mejores países del mundo en afecto positivo12. Valores medios por país 2006-2016.

\begin{tabular}{ccccc}
\hline Rank & Country & Number of observations & Positive affect & Negative affect \\
\hline 1 & Paraguay & 10995 & 0,842 & 0,222 \\
2 & Panamá & 11025 & 0,833 & 0,215 \\
3 & Costa Rica & 11006 & 0,829 & 0,279 \\
4 & Venezuela & 10994 & 0,824 & 0,243 \\
5 & El Salvador & 11008 & 0,818 & 0,319 \\
6 & Guatemala & 11045 & 0,812 & 0,297 \\
7 & Colombia & 10999 & 0,810 & 0,308 \\
8 & Ecuador & 11135 & 0,809 & 0,323 \\
9 & Canadá & 11325 & 0,804 & 0,257 \\
10 & Philippines & 12198 & 0,800 & 0,364 \\
11 & Iceland & 3131 & 0,799 & 0,217 \\
12 & Denmark & 10777 & 0,798 & 0,193 \\
13 & Honduras & 10991 & 0,797 & 0,273 \\
14 & Norway & 6010 & 0,797 & 0,208 \\
15 & Nicaragua & 11015 & 0,796 & 0,312 \\
\hline
\end{tabular}

Fuente: World Happiness Report (2018).

\section{CONCLUSIONES}

A partir de los anteriores planteamientos se puede enunciar, a modo de conclusión, que el análisis económico tiene que ser interdisciplinario. Es importante que diferentes áreas del conocimiento contribuyan a la comprensión de la complejidad de la acción humana, la interacción de la economía

\footnotetext{
11 El afecto positivo se define como el promedio de tres medidas de afecto positivo en GWP: felicidad, risa y disfrute, de la Encuesta Mundial Gallup (World Hapiness Report, 2018).

12 Afecto positivo medido como promedio simple de las siguientes cinco variables dicotómicas "día antes": sonrisa o reírse, aprender algo, tratado con respeto, disfrutar con experiencia y sentirse bien descansado.
} 
con otras disciplinas nos dotará de más herramientas para comprender los procesos de decisión de los individuos.

Desde la perspectiva evolutiva no es pertinente suponer que la acción humana sigue una lógica fundada en la maximización racional de la utilidad. Los seres humanos experimentan procesos externos e internos de adaptación, motivación y heurística, que pueden inspirarse mejor en un paradigma darwiniano, que en uno de corte newtoniano. La heterogeneidad del comportamiento de los sujetos no permite suponer escenarios teleológicos de optimización del bienestar.

El proceso cognitivo es complejo y está condicionado por factores diversos como la educación, las emociones y las interacciones sociales. Por otro lado, la adaptabilidad de la población depende de factores contextuales como el nivel de desigualdad y la temporalidad.

Las decisiones racionales del sujeto no se pueden entender por fuera de las emociones. Los avances en este campo son relativamente recientes, pero sorpresivamente rápidos. Se ha observado con ayuda de sistemas de imágenes cerebrales cómo el funcionamiento cerebral reacciona de manera diferente en individuos enfrentados a escenarios comunes.
La funcionalidad de estos postulados puede explorarse, por ejemplo, hacia el marketing y las ventas, debido al efecto dotación; una misma persona puede asignar dos valores diferentes a un producto dependiendo de si ese producto es suyo o no. En la práctica, esto provoca a menudo una diferencia en el valor $y$, por lo tanto, también en el precio ${ }^{13}$.

Algunos otros interrogantes que pueden responder estudios en este sentido son: ¿cómo funciona el cerebro de un corredor de bolsa?, ¿cómo funciona el cerebro de cualquier persona en distintos momentos de incertidumbre económica?, ¿cómo se generan las expectativas económicas a nivel individual y social?, ¿hay diferencias entre población urbana y rural en los procesos cognitivos de toma de decisión? o ¿cuáles son los efectos de los sentimientos morales y las emociones en los procesos de decisión?

Se espera que los hacedores de política consideren la importancia que tiene la "dimensión del comportamiento" que, finalmente, llevará a poner en tela de juicio los modelos de proyección actuales, a entender la racionalidad no como una verdad o un axioma, sino como una variable por considerar, y a asimilar el hecho de que no se pueden establecer equilibrios de mercado con una noción abstracta de emotividad, cuando esta condición es inherente al ser humano.

13 Ver más en: Hatton MacDonald et al. (2010), Cerda et al. (2012). 
Sin lugar a duda, las reflexiones contenidas en este artículo buscan contribuir al debate académico y generar nuevos espacios de exploración analítica sobre la importancia de asumir el estudio del comportamiento económico de los individuos desde otras disciplinas, cuyo aporte puede abrir amplios espacios de análisis y profundización del conocimiento.

\section{AGRADECIMIENTOS}

A los evaluadores anónimos de la Revista, por sus comentarios y sugerencias.

\section{DECLARACIÓN DE CONFLICTOS DE INTERESES}

Los autores declaran que no existe conflicto de intereses.

\section{FINANCIAMIENTO}

Este trabajo no tuvo ningún tipo de financiamiento institucional.

\section{CONTRIBUCIÓN DE LOS AUTORES}

Cindy Cifuentes Gómez: promotora de la iniciativa, a partir de su trabajo de grado en la Maestría de Economía (UPTC).

Siervo Tulio Delgado Ruiz: coautor y director del trabajo de grado en la Maestría en Economía (UPTC).

Jorge Iván González: coautor y revisor del artículo. 


\section{REFERENCIAS}

[1] Arrow, K. (1982). Risk Perception in Psychology and Economics. Economic Inquiry, 20(1), 1-9. https://doi.org/10.1111/j.1465-7295.1982.tb01138.x

[2] Bechara, A., Damasio, H., Tranel, D. \& Damasio, A. R. (1997). Deciding Advantageously Before Knowing the Advantageous Strategy. Science, 275(5304), 1293-1295. https://doi.org/10.1126/science.275.5304.1293

[3] Bergman, D. C. (1998). Evolutionary Aspects of Learning: The Interface Between Fitness and Action. In Proceedings of the 7th International Behavioral Ecology Congress.

[4] Bernoulli, D. (1954, January). Exposition of a New Theory on the Measurement of Risk. Econometrica, 22(1), 23-36. https://doi.org/10.2307/1909829

[5] Bjorklund, D. (2000). Children's Thinking: Developmental Function and Individual Differences. Wadsworth.

[6] Bjorklund, D. \& Pellegrini, A. (2002). The Origins of Human Nature: Evolutionary Developmental Psychology. American Psychological Association. https://doi. org/10.1037/10425-000

[7] Camerer, C. (1996). Rules for Experimenting in Psychology and Economics, and Why They Differ. In W. Albers et al. (eds), Understanding Strategic Interaction (pp. 313-327). Springer. https://doi.org/10.1007/978-3-642-60495-9_25

[8] Camerer, C., Loewenstein, G. \& Prelec, D. (2005). Neuroeconomics: How Neuroscience can Inform Economics. Journal of Economic Literature, 43(1), 9-64. https://oi.org/10.1257/0022051053737843

[9] Camerer, C. \& Lovallo, D. (1999). Overconfidence and Excess Entry: An Experimental Approach. The American Economic Review, 29(1), 306-318. https:// doi.org/10.1257/aer.89.1.306

[10] Cerda, A. A., García, L. Y., Ortega-Farías, S., \& M. Ubilla, A. (2012). Consumer Preferences and Willingness to Pay for Organic Apples. Ciencia e Investigación Agraria, 39(1), 47-59. https://doi.org/10.4067/S0718-16202012000100004

[11] Clapp, J. \& McDonnell, A. (2000). The Relationship of Perceptions of Alcohol Promotion and Peer Drinking Norms to Alcohol Problems Reported by College Students. Journal of College Student Development, 41, 19-26. 
[12] Damasio, A. (1994). Descartes' Error. Avon Books.

[13] Darwin, C. (1872). The Expression of the Emotions in Man and Animals. John Murray. https://doi.org/10.1037/10001-000

[14] De Martino, B., Kumaran, D., Seymour, B., \& Dolan, R. (2006). Frames, Biases, and Rational Decision-Making in The Human Brain. Science, 313, 684-687.

[15] Departamento Administrativo Nacional de Estadística -DANE-. (2017). Estadísticas de pobreza multidimensional del DANE. DANE.

[16] Dolan, P., Hallsworth, M., Halpern, D., King, D., Metcalfe, R. \& Vlaev, I. (2012, February). Influencing Behaviour: The Mindspace Way. Journal of Economic Psychology, 33(1), 264-277. https://doi.org/10.1016/j.joep.2011.10.009

[17] Flores, L. \& Ostroksy-Solís, F. (2008). Neuropsicología de lóbulos frontales, funciones ejecutivas y conducta humana. Revista Neuropsicología, Neuropsiquiatría y Neurociencias, 8(1), 47-58.

[18] Fodor, J. (2000). The Mind Doesn't Work That Way: The Scope and Limits of Computational Psychology. MIT Press. https://doi.org/10.7551/ mitpress/4627.001.0001

[19] Geary, D. C. \& Huffman, K. J. (2002). Brain and Cognitive Evolution: Forms of Modularity and Functions of Brain. Psychological Bulletin, 128(5), 667-698. https://doi.org/10.1037/0033-2909.128.5.667

[20] Genesove, D. \& Mayer, C. (2001). Loss Aversion and Seller Behavior: Evidence from the Housing Market. Quarterly Journal of Economics, 116(4), 1233-1260. https://doi.org/10.1162/003355301753265561

[21] Gilbert, T. (1989). Thinking Lightly About Others: Automatic Components of the Social Inference Process. In J. S. Uleman \& J. A. Bargh (eds.), Unintended Thought. Prentice-Hall, Englewood Cliffs.

[22] González, J. I. (2011). Psicología, biología y economía. Suma de Negocios, 2(2), 7-16.

[23] Hatton MacDonald, D., Morrison, M. \& Barnes, M. (2010). Willingness to Pay and Willingness to Accept Compensation for Changes in Urban Water Customer Service Standards. Water Resources Management, 24, 3145-3158. https://doi. org/10.1007/s11269-010-9599-7 
[24] Hernández-Blasi, C., Bering, J. \& Bjorklund, D. F. (2003). Psicología evolucionista del desarrollo: contemplando la ontogénesis humana desde los ojos del evolucionismo. Infancia y Aprendizaje, 26(3), 267-285. https://doi. org/10.1174/021037003322299043

[25] Hernández, I. (2010). Economía evolucionista y Darwin. Acta Biológica Colombiana.

[26] Hernández, I. (2011). Desigualdad, felicidad y la economía evolucionista. En V. A. Papini (ed.), Darwin y las ciencias del comportamiento (pp. 301-310). Universidad Nacional de Colombia.

[27] Hodgson, G. (1993). Institutional Economics: Surveying the 'Old' and the 'New'. Metroeconomica, 44(1),1-28. https://doi.org/10.1111/j.1467-999X.1993.tb00786.x

[28] Hogdson, G. (1997). The evolutionary and Non-Darwinian Economics of Joseph Schumpeter. Journal of Evolutionary Economics, 7, 131-145. https://doi. org/10.1007/s001910050038

[29] Kahneman, D. (1994). New Challenges to the Rationality Assumption. In New Challenges to the Rationality Assumption (pp. 18-36). https://www.jstor.org/ stable/40753012

[30] Kahneman, D. (2000). Evaluation by Moments: Past and Future. Cambridge University Press. https://doi.org/10.1017/CBO9780511803475.039

[31] Kahneman, D. (2002). Conference: The American Economic Review. American Psychologist, 93(5), 1449-1475. https://doi.org/10.1257/000282803322655392

[32] Kahneman, D. \& Frederick, S. (2005). A Model of Heuristic Judgment. In K. J. Holyoak \& R. G. Morrison (Eds.), The Cambridge Handbook of Thinking and Reasoning (pp. 267-293). Cambridge University Press.

[33] Kahneman, D. \& Lovallo, D. (1993). Timid Choices and Bold Forecasts: A Cognitive Perspective on Risk Taking. Management Science, 39(1), 17-31. https:// doi.org/10.1287/mnsc.39.1.17

[34] Kahneman, D. \& Tversky, A. (1979). Prospect Theory: An Analysis of Decisions Under Risk. Econometrica, 47(2), 313-327. https://doi.org/10.2307/1914185

[35] Kahneman, D. \& Tversky, A. (1982). The Psychology of Preferences. Scientific American, 246, 160-173. https://oi.org/10.1038/scientificamerican0182-160 
[36] Kahneman, D., Wakker, P. P. \& Sarin, R. (1997, May). Back to Bentham? Explorations of Experienced Utility. Quarterly Journal of Economics, 112(2), 375-405.

[37] Kusev, P., Van Schaik, P., Ayton, P., Dent, J., \& Chater, N. (2009). Exaggerated Risk: Prospect Theory and Probability Weighting in Risky Choice. Journal of Experimental Psychology: Learning, Memory and Cognition, 35(6), 1487-1505. https://oi.org/10.1037/a0017039

[38] Lagos, M. (2016). La confianza en América Latina. Latinobarómetro Opinión Pública Latinoamericana. www.latinobarometro.com

[39] La Rocca, S. M. (2003). Física, biología... ¿y después? Actas de las IX Jornadas de Epistemología de las Ciencias Económicas y IV Simposio de la Sociedad Iberoamericana de Metodología de la Economía. Facultad de Ciencias Económicas Universidad de Buenos Aires.

[40] Lerner, J. S. (1999). Accounting for the Effects of Accountability. Psychological Bulletin, 125(2). https://doi.org/10.1037/0033-2909.125.2.255

[41] Lichtenstein, S., Slovic, P., Fischhoff, B., Layman, M., \& Combs, B. (1978). Judged Frequency of Lethal Events. Journal of Experimental Psychology: Human Learning and Memory, 4(6), 551-578. https://doi.org/10.1037/0278-7393.4.6.551

[42] Loewenstein, G. \& Prelec, D. (1992). Anomalies in Intertemporal Choice: Evidence and an Interpretation. Quarterly Journal of Economics, 107(2), 573-597. https://oi.org/10.2307/2118482

[43] Loewenstein, G., Weber, E. U., \& Hsee, C. K. (2001). Risk as Feelings. Psychological Bulletin, 127(2), 267-286. https://doi.org/10.1037/0033-2909.127.2.267

[44] Mantzavinos, C., North, D. \& Shariq, S. (2004). Learning, Institutions, and Economic Performance. Perspectives on Politics, 2(1), 75-84. https://doi. org $/ 10.1017 /$ S1537592704000635

[45] Masera, G. (2013, dic.). Evolucionismo histórico y biológico. En el debate económico contemporáneo. Filosofía de la Economía,1(2), 69-87.

[46] McClure, S. M., Ericson, K. M., Laibson, D. I., Loewenstein, G., \& Cohen, J. D. (2007). Time Discounting for Primary Rewards. Journal of Neuroscience, 27, 5796-5804. 
[47] McClure, S. M., Laibson, D. I., Loewenstein, G., \& Cohen, J. D. (2004). Separate Neural Systems Value Immediate and Delayed Monetary Rewards. Science, 306, 503-507.

[48] Montoya, M. I. (2010). Situación de la competitividad de las pyme en Colombia: elementos actuales y retos. Agronomía Colombiana, 28(1), 107-117.

[49] Ng, Y.-K. (1996, April 21). Complex Niches Favour Rational Species. Journal of Theoretical Biology, 179(4), 303-311. https://doi.org/10.1006/jtbi.1996.0069

[50] Oswald, A. (1997). Happiness and Economic Performance. Economic, 107(445). https://doi.org/10.1111/j.1468-0297.1997.tb00085.x

[51] Posner, M. I. \& Keele, S. W. (1968). On the Genesis of Abstract Ideas. Journal of Experimental Psychology, 77(3), 353-363. https://doi.org/10.1037/h0025953

[52] Robson, A. (2002). Evolution and Human Nature. The Journal of Economic Perspectives,16(2), 89-106. https://doi.org/10.1257/0895330027274

[53] Robson, A. (2003). The Biological Basis of Economic Behaviour. Journal of Economic Literature, 39, 11-33. https://doi.org/10.1257/jel.39.1.11

[54] Robson, C. (2011). Real World Research: A Resource for Social-Scientists and Practitioner-Researchers. Blackwell Publishing.

[55] Rogers, A. (1994). Evolution of Time Preference by Natural Selection. American Economic Review, 84(3), 460-481.

[56] Rosch, E. \& Mervis, C. (1975). Family Resemblances: Studies in the Internal Structure of Categories. Cognitive Psychology, 7(4), 573-605. https://doi. org/10.1016/0010-0285(75)90024-9

[57] Ross, L. \& Nisbett, R. (1991). The Person and the Situation. McGraw-Hill.

[58] Rottenstreich, Y. \& Hsee, C. (2001). Money, Kisses and Electric Shocks: On the Affective Psychology of Risk. Psychological Science, 12(3), 185-190. https://doi. org/10.1111/1467-9280.00334

[59] Schaap, M. K. (2008). Effect of Nationwide Tobacco Control Policies on Smoking Cessation in High and Low Educated Groups in 18 European Countries. Tobacco Control, 17(4), 248-255. https://doi.org/10.1136/tc.2007.024265

[60] Scitovsky, T. (1992). The Joyless Economy. Oxford University Press. 
[61] Simon, H. (1986). Rationality in Psychology and Economics. The Journal of Business, 59(4), Part 2: The Behavioral Foundations of Economic Theory, S209-S224. https://doi.org/10.1086/296363

[62] Slovic, P. M. (2002). Influence of Affective Processes on Toxicologists' Judgments of Risk. Unpublished Study.

[63] Stanovich, K. E. \& West, R. F. (2002). Individual differences in reasoning: Implications for the rationality debate? In T. Gilovich, D. Griffin\& D. Kahneman (Eds.), Heuristics \& Biases: The Psychology of Intuitive Judgment (pp. 421-440). Cambridge University Press. https://doi.org/10.1017/CBO9780511808098.026

[64] Sternberg, R. (1997). The Concept of Intelligence and Its Role in Lifelong Learning and Success. American Psychologist, 52(10), 1030-1037. https://doi. org/10.1037/0003-066X.52.10.1030

[65] Strotz, R. (1956). Myopia and Inconsistency in Dynamic Utility Maximization. Review of Economic Studies, 23(3),165-180. https://doi.org/10.2307/2295722

[66] Thaler, R. (1980). Toward a Positive Theory of Consumer Choice. Journal of Economic Behavior and Organization, 1(1), 39-60. https://doi. org/10.1016/0167-2681(80)90051-7

[67] Thaler, R. (1985). Mental Accounting and Consumer Choice. Marketing Science, 4, 199-214. https://doi.org/10.1287/mksc.4.3.199

[68] Thaler, R. (1999). Mental Accounting Matters. Journal of Behavioral Decision Making, 12, 183-206. https://doi.org/10.1002/(SICI)1099-0771(199909)12:3<183::A ID-BDM318>3.0.CO;2-F

[69] Thaler. R. \& Kahneman, D. (1991). Economic Analysis and The Psychology of Utility: Applications to Compensation Policy. American Economic Review, 81(2), 341-352.

[70] Thaler, R. \& Sunstein, C. (2017). Nudge, Improving Decisions About Health, Wealth, and Happiness. Penguin Random House.

[71] The Nobel Prize. (2021). Richard H. Thaler Facts. https://www.nobelprize.org/ prizes/economic-sciences/2017/thaler/facts/

[72] The Nobel Prize. (2021). Daniel Kahneman Facts. https://www.nobelprize.org/ prizes/economic-sciences/2002/kahneman/facts/ 
[73] Tom, S., Fox, C., Trepel, C., \& Poldrack, R. (2007). The Neural Basis of Loss Aversion in Decision-Making Under Risk. Science, 315(5811), 515-518.

[74] Tremblay, L., Hollerman, J. \& Schultz, W. (1998). Modifications of Reward Expectation-Related Neuronal Activity During Learning in Primate Striatum. Journal of Neurophysiology, 80(2), 964-977. https://oi.org/10.1152/ jn.1998.80.2.964

[75] Tversky, A. \& Kahneman, D. (1981). The Framing of Decisions and the Psychology of the Choice. Science, 211(4481), 453-458. https://doi.org/10.1126/science.7455683

[76] Tversky, A. \& Kahneman, D. (1986). Rational Choice and the Framing of Decisions. Journal of Business, 251-278. https://doi.org/10.1086/296365

[77] Tversky, A. \& Kahneman, D. (1991). Loss Aversion in Riskless Choice: A Reference-Dependent Model. Quarterly Journal of Economics, 106(4),1039-1061. https://doi.org/10.2307/2937956

[78] Watanabe, M. \& Sakagami, M. (2007). Integration of Cognitive and Motivational Context Information in the Primate Prefrontal Cortex. Cerebral Cortex, 17, 101109. https://doi.org/10.1093/cercor/bhm067

[79] Witt, U. (2003). The Evolving Economy: Essays on the Evolutionary Approach to Economics. Edward Elgar.

[80] Word Happiness Report. (2017). Report. https://worldhappiness.report/ed/2017/

[81] Word Happiness Report. (2018). Report. https://worldhappiness.report/ed/2018/ 